\title{
Technology Factors of Improving the Performance Properties of Anticorrosive Protective Coatings for Safety Enhancement of Coal Enterprises
}

\author{
Tatiana Cherkasova ${ }^{1, *}$, Yelizaveta Cherkasova $^{1}$, and Anastasia Tikhomirova ${ }^{1}$ \\ ${ }^{1}$ T.F. Gorbachev Kuzbass State Technical University, 650000, Russian Federation, Department of \\ Chemistry, Technology of Inorganic Substances and Nanomaterials, 650000 Kemerovo, 28 Vesennya \\ st., Russian Federation
}

\begin{abstract}
Corrosion, aging, wear of the basic production assets of the of the Kuznetsk Basin coal industry enterprises lead to huge losses of natural resources and material supplies; labor costs being increased. Most failures, breakdowns, malfunctions at industrial facilities, except for cases of misuse, are caused by the above mentioned factors. Therefore, the primary task is to develop methods for protecting equipment from corrosion. In this paper, the measures to increase the longevity of polymer-silicate mastic protective coatings by studying and optimizing the group of internal properties of polymer-silicates that determine the properties of the coating as a whole are proposed.
\end{abstract}

\section{Introductuion}

The main task of protective measures is to achieve the maximum possible service life of fixed production assets with minimal material and labor costs. When developing, manufacturing and operating in various modes, it is necessary to achieve such an interaction of internal and external factors causing corrosion, under which the maximum service life with minimal costs for protection, operation and repair is achieved.

Restoration of corrosion protection for the operating enterprises is often difficult, therefore it is performed poorly. The damage caused by corrosion of structures and buildings is supplemented by the costs of their repair and restoration, as well as the work stoppage costs. In this regard, the problem of increasing the reliability and durability of buildings, structures, equipment and protecting them from corrosion becomes extremely important.

In the anticorrosive methods, acid-resistant concrete, mortars and liquid glass putty have been known for a long time. They are resistant to the action of concentrated acids, including hot-laid ones. However, these materials are limited in water resistance, weak acid media and completely non-resistant to the action of alkalis. The main disadvantage of such coatings is high through porosity, which, depending on the composition, can reach $10-20 \%$,

\footnotetext{
*Corresponding author: ctg.htnv@,kuzstu.ru
} 
which leads to high permeability. For these reasons, the use of such coatings in our country was small [1-3].

The improvement of the polymer-silicate protective coating technology posed a number of problems dealing with producing new compositions and modifying compositions taking into account the specifics of their use and the formation of thin-layer plasters; definition of the relationship between technological and formulation parameters; detection of reserves of progressive liquid-glass pargeting; increase of the protective properties of thin-layer polymer-silicate coatings with technological methods. To solve the set task, the effect of various modifiers acting independently and in various combinations on the qualitative and quantitative level of the limiting state of protective coating, as well as on the construction with protective coating [4-8] was studied on the basis on the results of scientific and technical analysis of research and practical developments in this field.

Modifiers used for liquid glass silicate acid-resistant materials can be varied both in nature and in the mechanism of their effect on the components of acid-resistant concrete, mortars, including: caulking or dispersing (furan, phenolic and other resins), water binding (NCO compounds, etc.), densifying (oligoesters), retarding hardening (sulfonol, organosilicon liquids), colmatizing (rosin, sulfur and etc.), plasticizing, etc.

To obtain a complex effect, the modifiers can be admixed alone or in various combinations. As a result of the performed studies, it was possible to significantly improve the technological properties, water resistance and resistance to alkali solutions of known polymer silicas, and also to increase the adhesion of polymer silicate plasters to cement concrete [9-11].

The use of polymer-silicate compositions as protective coatings opens up a wide perspective in a significant increase in the service life of technological equipment and structures. The cost of the components of the polymer-silicate coatings is 2-8 times lower than that of the currently used in these cases at the moment, and taking into account the service life and operating costs, they are more effective 40-100 times [12-15].

\section{Materials and methods}

The experiment was based on the known results of many years of research related to the change in the mechanism for curing the liquid-glass binder. The basis of these studies is that the alkali in the system is not neutralized to form the fluffy precipitate, but enters into a chemical reaction with a thermodynamically unstable component, tetrahydrosilicate. The curing of polymer silicas according to this principle determines the thermodynamic stability of the binder, the increase in strength (2-3 times), water and alkali resistance. At stoichiometric ratio of liquid glass and active filler, the polymer silicates become acid-, water- and alkaline-impermeable.

In order to study the influence of the components and optimize the polymer-silicate composition, the following basic and modifying (structuring, densifying, plasticizing) components were selected to improve chemical resistance, water resistance, impermeability, strength properties.

The following materials were used:

- liquid sodium glass (State Standard 13078-81) with a density of $1.38-1.42 \mathrm{~g} / \mathrm{cm}^{3}$ with a silicate module of 2.6 as a binder for polymer-silicate compounding;

- sodium hexafluorosilicate (Specification Requirements 113-08-587-86) with a basic product level of not less than $93 \%$ as a curing agent. The curing agent moisture is $1 \%$. Grinding fineness (State Standard 3102-76) corresponds to the $100 \%$ passage through a 008 sieve;

- a floured filler is an acid-resistant effusive volcanic rock (andesite, diabase, etc) powder according to Specification Requirements 6-10- 101-81. Acid resistance is not less 
than $97-98 \%$ according to State Standard 473.1- 81. Humidity of the filler is not more than $1 \%$. Grinding fineness corresponds to the more than $90 \%$ passage through a 0315 sieve.

- a washed mason sand (State Standard 8736-96) with a content of dust-prominent, muddy and clayey parts of not more than $1 \%$ was used as a fine filler. The grain composition was in the range of $0.15-2.5 \mathrm{~mm}$ and corresponded to the requirements of State Standard 10268-80. The absence of admixtures of limestone, dolomite, and metallic inclusions in the filler was verified by the hydrochloric acid solution sample. Acid resistance of sand is not less than $96 \%$;

- a sodium methylsiliconeate $(15 \%$ aqueous alcohol solution, Organic Silicon Compound-11 (Specification Requirements 6-02-696-76)) was used as a hydrophobizing, modifying modifier, increasing the pot-life of the mixture;

- a waterproofing isocyanates polyisocyanate polyur BT, grade B (Specification Requirements 2224-152-04691277-96) with a mass fraction of isocyanate groups of $31.1 \%$ was used as a densifying alkali-resistant polymer agent;

- a superplasticizer-diluent C-3 (Specification Requirements 6-14-625-80) was used as a plasticizing agent increasing the workability of the polymer-silicate mixture.

When selecting the quantitative content of the constituent components and the optimal ratio between them, we proceeded from the conditions of the lowest liquid glass consumption, the good workability observance, and the pot-life increase.

\section{Key results}

Due to the complexity of the process of interaction of liquid glasses with the components of polymer-silicate compounds, the quality of the protective coating depends very strongly on the rate and duration of mixing, and not only the concentration and ratio of reagents, temperature, humidity, etc. A correlation between the optimal dosage of structure-forming and plasticizers agents, on the one hand, and the rate and duration of mixing, on the other hand, on the physical, chemical and technological characteristics of concrete mixtures. In particular, it was found that, at high mixing speed, 15 minutes after the start of mixing in the concrete mixer truck, the concrete mixes become practically uniform. The gas content reaches a maximum value after 30 minutes and with further mixing (up to 1.5 hours) it remains at a constant level, providing high frost resistance to structures. This provision is extremely important for our studies, since the value of "gas content" consists of two components: the amount of released gas (hydrogen) and air, which is always involved in the concrete mixture while mixing it. As the density of the mixture increases, the volume of entrained air decreases somewhat, but the workability of the composition is sufficiently high due to the optimum amount of plasticizer. Thus, it is possible to increase the content of the organosilicon hydrophobizing modifier, which prolongs the pot-life of the mixture (and hence the reaction time of the reactants) without exceeding the critical level of the gas content, and thereby form high modulus malohydrated hydrosilicate bonds having acid- and alkali resistance due to stoichiometric interaction of sodium hexafluorosilicate with sodium silicate, dispersed polyisocyanates.

Therefore, we selected and studied the effect of each agent on physical and chemical properties of the compositions, the rate of mixing of which significantly exceeded the traditionally established ones. Special attention was paid to the duration of setting (pot-life) of mortars, determined by the time (in minutes) that has elapsed since the mortar mixing to the point when it was not distributable on the horizontal surface (beginning of curing).

Compositions 1, 2 and 3 were made as controls.

Composition 1: a silicate mortar of a conventionally used composition, which is shown in Table 1. 
Table 1. The conventional composition of the silicate solution.

\begin{tabular}{|c|c|c|c|c|}
\hline component & $\begin{array}{r}\text { sodium silicate, } \\
\boldsymbol{\rho}=\mathbf{1 , 4 0} \mathbf{~ g / \mathbf { c m } ^ { 3 }}\end{array}$ & $\begin{array}{c}\text { curing agent, } \\
\text { fluorosilicate }\end{array}$ & andesite powder & sand \\
\hline amount, pts. wt. & 100 & 15 & 130 & 140 \\
\hline
\end{tabular}

Its setting duration is known and is 5 hours; the duration of complete curing is 8 hours, respectively. The mortar should be mixed mechanically in mortar mixers. First, a required amount of sodium silicate, and then a mixture of filler and sodium hexafluorosilicate, as a curing agent, is fed into the mortar mixer while agitating continuously. In the absence of a ready mixture of dry constituents, the filler and curing agent are added successively. It is also possible to reverse the feed order. Rotational speed of the movable object of industrial mortar mixer is $30-32 \mathrm{rpm}$. The agitating time is $2-3 \mathrm{~min}$.

Composition 2: a polyisocyanate-modified polymer-silicate mortar developed by Concrete and Reinforced Concrete Research Institute Gosstroy of the USSR State Committee for Construction and included in the regulatory and technical documentation is presented in Table 2:

The composition mixing mode is similar to the mixing mode of composition 1 with restrictions on the duration of mixing. The total duration of mixing should not exceed $130 \mathrm{~s}$, taking into account the discharge of not more than 3 minutes; the duration of mixing of sodium silicate with additives - $10 \mathrm{~s}$, with fillers - 30-60 s, with curing agent - $30 \mathrm{~s}$.

Composition 3: As a third control, the mortar was prepared according to formula of Composition 2; however, the mixing mode was changed. The duration of mixing operation and the rate of mixing of the components were increased so that the total mixing time was 15 minutes at a rotational speed of the mixer agitator of at least $600 \mathrm{rpm}$. In this case, the order of feeding the components corresponded to the order of feeding the components of composition 1. The duration of mixing of modified sodium silicate is 2 minutes, with fillers - 3 minutes, with curing agent - 10 minutes.

Table 2. Composition of a polyisocyanate-modified polymer-silicate mortar.

\begin{tabular}{|c|c|c|c|c|c|c|c|}
\hline component & $\begin{array}{c}\text { sodium } \\
\text { silicate, } \\
\rho=1,40 \\
\mathrm{~g} / \mathrm{cm}^{3}\end{array}$ & 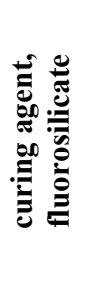 & 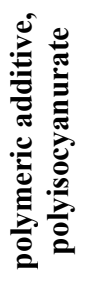 & 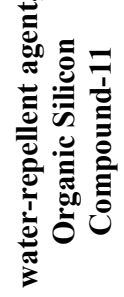 & 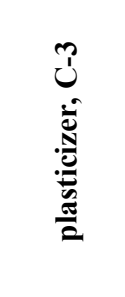 & $\begin{array}{l}\text { andesite } \\
\text { powder }\end{array}$ & sand \\
\hline $\begin{array}{l}\text { amount, } \\
\text { pts. wt. }\end{array}$ & 19,0 & 2,8 & 5,7 & $0,01-0,2$ & $0,01-0,2$ & 23,4 & 49,0 \\
\hline
\end{tabular}

Sodium liquid glass of the required density was produced by diluting industrial glass with a density of $1.54-1.55 \mathrm{~g} / \mathrm{cm}^{3}$ with water modified by the additives added in advance in the calculated amount. The amount of water to achieve an operating density of $1.38 \mathrm{~g} / \mathrm{cm}^{3}$ was determined by the formula:

$$
\rho_{l} V_{l}=\rho_{2} V_{2},
$$

where $\rho_{l}$ - initial density of liquid glass, $\mathrm{g} / \mathrm{cm}^{3}$,

$V_{l}$ - amount of liquid glass of density $\rho_{l}, 1$,

$\rho_{2}-$ required liquid glass density, $\mathrm{g} / \mathrm{cm}^{3}$, 
$V_{2}$ - calculated amount of liquid glass of density $\rho_{2} ., 1$.

The amount of water necessary for the dilution of industrial liquid glass is determined by the difference between the volumes of diluted and industrial liquid glass:

$$
\text { Vwater }=V_{2}-V_{l} \text {, }
$$

Dried dry components - sand, andesite powder, sodium hexafluorosilicate and plasticizer were thoroughly mixed. Diluted liquid glass was separately mixed with polyisocyanate and hydrophobizing liquid Organic Silicon Compound-11 (sodium methylsiliconeate). Then, sodium silicate with modifiers was introduced into a ready dry mixture while agitating. The liquid-glass composition having been mixed; the mixture was poured into metal detachable molds coated with a thin layer of lubricant to make samples in the form of $(70.7 \times 70.7 \times 70.7) \mathrm{mm}$ size cubes. Also, samples in the form of pellets with a diameter of $\sim 75 \mathrm{~mm}$ in various thicknesses were made.

The samples made in accordance with State Standard 5802-86 were cured in molds for 24 hours. After a 24-hour curing, the samples were cured to provide the strength development for 10 days in air-dry conditions at room temperature and relative humidity of not more than $75 \%$.

The duration of setting was checked on the samples made in the form of pellets. The appearance of salt efflorescences, cracks and other unacceptable defects was also estimated in these samples.

As a result of this preliminary experiment, carried out to obtain the initial data for further optimization of the composition and technological parameters, the correspondence of the obtained data to the regulatory values of the duration of setting, curing and the basic physical and chemical properties, which are presented in Table 3, was confirmed.

Table 3. The basic technical, physical and chemical properties of compositions 1-3.

\begin{tabular}{|c|c|c|c|c|c|c|c|c|}
\hline \begin{tabular}{|l|} 
composition \\
\end{tabular} & 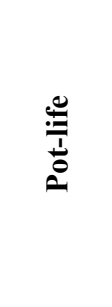 & 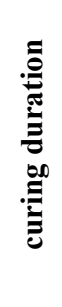 & 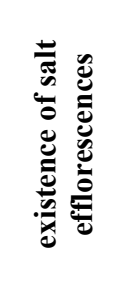 & 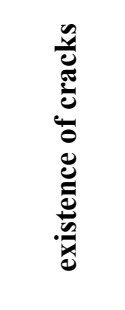 & $\stackrel{\varrho}{\Xi}$ & 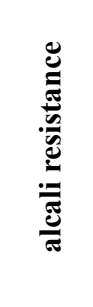 & 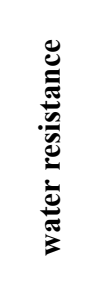 & 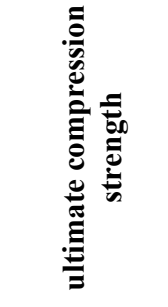 \\
\hline $\begin{array}{l}1 \\
2 \\
3\end{array}$ & $\begin{array}{c}5 \mathrm{~h} \\
40 \mathrm{~min} \\
60 \mathrm{~min}\end{array}$ & $\begin{array}{l}8 \mathrm{~h} \\
4 \mathrm{~h} \\
4 \mathrm{~h}\end{array}$ & $\begin{array}{c}\text { yes } \\
\text { yes } \\
\text { valueless }\end{array}$ & $\begin{array}{c}\text { yes } \\
\text { valueless } \\
\text { no }\end{array}$ & $\begin{array}{c}\text { yes } \\
\text { valueless } \\
\text { no }\end{array}$ & $\begin{array}{l}\text { failure } \\
\text { failure } \\
2 \text { часа }\end{array}$ & $\begin{array}{c}\text { failure } \\
0,8 \\
1,0\end{array}$ & $\begin{array}{l}\text { corresponds } \\
\text { corresponds } \\
\text { corresponds }\end{array}$ \\
\hline
\end{tabular}

It can be seen from the table that an increase in the rate and duration of mixing led to an improvement in all control parameters. However, the pot-life of the composition remained unsatisfactorily small.

The analysis of the process of curing composition 3 showed that the standard amount of sodium methylsiliconate, which acts as hydrophobizing and air-entraining agent, increasing a pot-life, is not sufficient to achieve a setting time that would ensure the normal operation mode at the construction site, that is, not less than 2 hours.

Therefore, the aim of the second stage of the experiment was to study the possibility of achieving the optimal pot-life by increasing the content of sodium methyl siliconate without reducing the strength properties of the cured mortar. The fulfillment of this condition is fundamental, since the agent slowing the setting process is simultaneously air-entraining. The excess air intake, size and quantity of air bubbles have a negative effect on the pore 
structure and density of the mortar, and, consequently, on its strength, frost resistance, permeability and other qualities.

The study of the polymer-silicate mortar of composition 3 with different contents of sodium methyl siliconate showed that the maximum pot-life of 2 hours is reached with the content of this air-entraining additive from $3 \%$ by weight of liquid glass. With an increase in the amount of more than 3\%, the pot-life practically did not increase, but the structure of the cured mortar became unacceptably porous due to the growing air entrainment. In addition, the pores were connected in through channels, and this immediately made the composition inadequate to the requirement of water impermeability.

Taking into account that the plasticizers very slightly reducing the viscosity of the unfilled liquid glass, at the same time significantly increase the workability of the mortar, it seemed promising to estimate the effect of increasing in the amount of plasticizing agentplasticizer C-3 on the structure (porosity and density) of composition 3 . As a result of the experiment it was found that an increase in this additive from 3 to $5 \%$ (by weight of liquid glass) significantly reduced the air entrainment (pore formation) of the mortar. This can be explained by the fact that there is a strong dilution of the mortar and the air outlet is facilitated. It was established that with the simultaneous increase in the content of airentraining (Organic Silicon Compound-11) and plasticizing agents in amounts of 3\% each by weight of liquid glass, the pot-life of composition 3 increased to $\sim 2.5$ hours. The completion time of the mortar curing did not change and still corresponded to 4 hours.

When assessing the appearance and the cleavage surface of the samples with the help of the 30-power MBS-7 microscope, cracks, large pores, connecting channels, and also salt efflorescences were not detected.

Further tests of the physical and chemical properties did not reveal the reduction in strength and resistance of the polymer-silicate mortar of the optimized composition 4 during the control measurements.

\section{Conclusions}

As a result of the optimized composition 4 (containing 3\% Organic Silicon Compound-11 and C-3 by weight of liquid glass) pot-life study, the characteristic features of not only the simultaneous influence of the components manifesting a synergistic effect, but, to a greater extent, as well as of special technological mortar mixing methods on the increase in workability and remolding effort $(\sim 15 \%)$ and simultaneously (almost 2.5 times) a pot-life.

In the course of the work, the peculiarities of the influence of the properties of polymer silicates on the parameters of the technological coating were revealed, the relationship between the methods of mixing, the compositions of the polymer silicates and such parameters as setting and curing time was established.

It has been found that the use of polymer-silicate compounds provides the specified durability of fixed production assets of industrial enterprises and is an effective method of corrosion protection.

\section{References}

1. R.G. Buchheit, J. Electrochem. Soc,. 142, 3994 (1995)

2. T.P. Chou, C. Chandrasekaran, G.Z. Cao, J. Sol. Gel. Sci. Technol., 26, 321 (2003)

3. R.B. Figueira, C.J.R. Silva, E.V. Pereira, J. Coat. Technol. Res., 12, 1 (2014)

4. P. Rodič, J. Iskra, I. Milošev, J. Sol. Gel. Sci. Technol., 70, 90 (2014)

5. S. Zheng, J. Li, J Sol. Gel. Sci. Technol, 54, 174 (2010) 
6. J. Wen, G.L. Wilkes, Chem. Mater., 8,1667 (1996)

7. H. Wang, R. Akid, Corros. Sci., 50, 1142 (2008)

8. T.L. Metroke, R.L. Parkhill, E.T. Knobbe, Prog. Org. Coat., 4, 98 (2001)

9. N. Pirhady Tavandashti, S. Sanjabi, T. Mater, Corros., 62, 411 (2011)

10. G. Schottner, Chem. Mater., 13, 3422 (2001)

11. H. Wang, R. Akid, M. Gobara, Corros. Sci., 52, 2565 (2010)

12. P. Innocenzi, G. Brusatin, F. Babonneau, Chem. Mater., 12, 3726 (2000)

13. R. N. Peres, E. S. F. Cardoso, M. F. Montemor, H.G. Melo, A.V. Benedetti, P.H. Suegama, Surf. Coat. Technol. (Part B), 303, 372 (2016)

14. F. Khelifa, M-E. Druart, Y. Habibi, F. Bénard, P. Leclère, M. Olivier, P. Dubois, Prog. Org. Coat., 76, 900 (2013)

15. B. Volarič, I. Corros, Eng. Sci. Technol., 52, 201 (2017) 